\title{
Botulinum Toxin Injections as a Treatment of Postoperative Chronic Neck Pain Secondary to Cervical Spine Surgery
}

\author{
James Meiling1, (1) Brandon Barndt² George Raum ${ }^{3, \text { (일 }}$ David Schulze ${ }^{1}$ \\ ${ }^{1}$ Department of Internal Medicine, Medical City Weatherford, \\ Weatherford, Texas, United States \\ 2Department of Internal Medicine, Mercy Catholic Medical Center, \\ Philadelphia, Pennsylvania, United States \\ ${ }^{3}$ Philadelphia College of Osteopathic Medicine, Philadelphia, \\ Pennsylvania, United States \\ Address for correspondence James Meiling, DO, 5009 Lincoln Oaks \\ Drive South, Apt 104, Fort Worth, TX 76132, United States \\ (e-mail: james.bryan.meiling@gmail.com). \\ Indian J Neurosurg:2020;9:122-126
}

\begin{abstract}
Keywords

- postoperative neck pain

- botulinum toxin

- cervical spine surgery

Postoperative neck pain is a relatively common consequence of cervical spine surgeries such as anterior cervical discectomy with fusion and laminoplasty. Botulinum toxin injections are a relatively new treatment option to target muscle spasms, a possible cause of postoperative neck pain. This systematic review aims to evaluate the current literature highlighting the utilization of botulinum toxin for treating postoperative neck pain. The literature search resulted in one preliminary case-control trial, one case series and one case report. The use of Botox in these studies had promising benefits on postoperative neck pain, by reducing visual analog scores and improving objective measures of muscle tone such as cervical lordosis angle. While not seen in these studies, the use of botulinum toxin injections into the muscles of the region of the head, neck, and shoulder is not without risks such as focal muscle loss, joint instability, and respiratory and swallowing difficulty. With this in mind, while botulinum toxin may be a promising treatment for these patients, further well-designed studies are needed to further assess the treatments efficacy and safety in this patient population.
\end{abstract}

\section{Introduction}

A frequent, yet seldom studied, consequence of cervical spine surgery is postoperative neck pain, ${ }^{1,2}$ which has been found to affect up to $19 \%$ of patients after anterior cervical discectomy with fusion (ACDF) and up to $60 \%$ after laminoplasty. ${ }^{2}$ Physicians are still unsure of the precise etiologies of this postoperative neck pain, but it can be assumed that they most likely are related to the surgical disruption of muscular and ligamentous tissue, resulting in chronic muscle spasms. ${ }^{2}$

One newer approach for the alleviation of postoperative chronic neck pain is the utilization of botulinum toxin (BTX) injections into the cervical musculature, providing a relaxation of tight, spasming or tonic muscles. ${ }^{3}$ With the added stability of the neck due to vertebral fusion hardware, are BTX injections a possible treatment? BTX injections have previously been applied in the head and neck region for chronic pain relief in conditions such as blepharospasm, trigeminal neuralgia, facial pain, cervicogenic headaches, cervical dystonia, chronic neck pain, temporomandibular disorders, and migraines, ${ }^{3-5}$ but they have not been frequently utilized in regard to postoperative chronic neck pain. The purpose of this systematic review of the current literature is to summarize all of the pertinent studies that have been published regarding BTX injections as a treatment option for postoperative chronic neck pain secondary to cervical spine surgery.

\section{Methods}

A literature review was performed in September 2019 using the PUBMED and MEDLINE databases, using specific 
keywords and phrases, including "botulinum toxin," "postoperative," "neck pain," and "cervical spine surgery." Inclusion criteria required the articles to discuss using BTX for chronic neck pain after the patient had received cervical spine surgery. Articles discussing other types of injections for chronic neck pain, BTX injections for chronic neck pain without previous cervical spine surgery, or BTX injections for other craniofacial pathologies were excluded. The reference lists in each of these selected articles were then examined to ensure completeness in the overview of the current literature.

\section{Results}

A literature review was performed of the PUBMED and MEDLINE databases using the following search terms: (BTX OR Botox OR onabotulinum) AND (postop OR postoperative OR postsurgical) AND (cervical OR vertebral OR vertebrae OR spine OR spinal OR neck) AND (pain). The search resulted in many abstracts, which were then reviewed for applicability of this systematic review's purpose. Inclusion criteria included articles pertaining to the treatment of postoperative neck pain with BTX. Articles were not included if the neck pain was not postoperative, BTX was not utilized, BTX was used in areas other than the neck, or BTX was used for other pathologies, such as migraines. Asa result of the online literature review, three articles were located that fit the overall purpose of the author's systematic review-preliminary case-control trial, case series, and case report. The results of these studies, and a look at BTX, both its use perioperatively and its complications, are discussed in the main body of this article.

\section{Discussion}

\section{Botulinum Toxin Injections}

Clostridium botulinum, the bacteria that produces BTX, was first discovered in the 19th century. BTX, itself, was not discovered until 1936, and it was not until the 1970s that BTX was used in the study of neuromusculoskeletal medicine, primarily in animal studies. The first use of the toxin as a therapeutic modality was for strabismus, a vision impairment involving malalignment of the eyes, in the 1980s. ${ }^{6}$ From that time, use of BTX expanded to other neuromuscular disorders including dystonia, muscular spasms, spasticity (secondary to stroke, spinal cord injury, multiple sclerosis, and cerebral palsy), as well as chronic migraines. ${ }^{7}$ There are multiple serotypes of BTX, named from A to G. Botulinum toxin serotype-A (BTX-A) is the most commonly used serotype in clinical practice, while BTX-B and BTX-F have also been used but are less potent and have a shorter duration of therapeutic effect. ${ }^{6}$

BTX is synthesized as an inactive polypeptide that is then cleaved by endogenous proteases or exogenous proteases, such as trypsin. Trypsin is commonly used in the commercial preparation of BTX. Cleavage of the polypeptide is important for the production of the therapeutic toxin, as the presence of the uncleaved protein may be implicated in the production of neutralizing antibodies. ${ }^{6}$

The neurotoxin's action begins with binding to receptors on the membranes of cholinergic neurons, which causes internalization of the receptor via endocytosis. ${ }^{3,6}$ Once internalized, BTX destabilizes the SNARE protein complex. The SNARE protein complex is responsible for the fusion of acetylcholine-containing vesicles with the synaptic membrane for neurotransmitter release. Without the SNARE-mediated fusion of these neurotransmitter-containing vesicles with the neuronal membrane, acetylcholine cannot be released into the neuromuscular junction to cause muscle contraction; thus, the muscle stays relaxed. BTX modulates muscular activity in a dose-dependent fashion. ${ }^{6}$

The effect of BTX begins to wane at 4 weeks, as new SNARE proteins are produced by the neuron and the exocytosis of acetylcholine into the neuromuscular junction increases in density. Axonal sprouting initially regains nerve conduction, with elongation of the neuronal endplate. This leads the clinical chemodenervation of BTX to have an effect for 12 to 16 weeks. $^{6}$

\section{Perioperative Use of Botulinum Toxin to Control Cervical Dystonias}

Perioperatively, BTX has a clinical use during cervical spine surgery as a means to immobilize the neck in patients with tics and cervical dystonia (also known as spasmodic torticollis). ${ }^{8-12}$ Cervical dystonia is a cause of degenerative cervical spine disease. Over time, the chronic, unnatural position of the cervical vertebrae from dystonia can result in bone spur formation and foraminal narrowing, leading to myelopathy and radiculopathy. Patients with these conditions will often undergo surgical correction, such as ACDF, requiring postoperative immobilization ranging from weeks to months. Although immobilization is primarily achieved through the use of a halo brace, chronic repetitive neck motions may make adequate postoperative immobilization difficult, delaying the healing period and decreasing that patient's tolerance of halo brace use. To ameliorate this issue, BTX has been used as an adjunct to surgery to relax cervical musculature, thereby promoting immobilization by inhibiting these repetitive neck movements and reinforcing the cervical fixation..$^{8-14}$ The patient received BTX injections prior to their surgery, enabling the cervical dystonias to relax; this allowed the surgeon to install the proper cervical hardware and a postoperative halo brace. ${ }^{13}$ BTX injections, by itself, do not completely resolve the motions, in that some patients still have remnants of cervical dystonia, but the injections can help ease the motions prior, during, or after surgery. ${ }^{11}$ It was concluded that BTX injections were a relatively safe procedure that enabled greater postoperative immobilization tolerance in patients with cervical dystonia. ${ }^{8-10,12,13}$

\section{Use of Botulinum Toxin to Treat Chronic Postoperative Neck Pain}

Patients receive vertebral hardware when undergoing cervical fusions, with the aim of providing increased stability of the 
cervical spine. As a consequence, patients have a decreased range of motion of the neck postoperatively, which often leads to chronic contractions of the neck musculature. These ever-contracted muscles can produce chronic neck pain. Logically, if BTX injections can be used to decrease spasticity or dystonic muscles, then the same principle should be able to be applied for chronically tightened muscles of the neck. BTX injections directly into key muscles of the cervical musculature should relax the spasms. With relaxation of the muscles should come a consequent decrease in the associated neck pain. ${ }^{3}$

The mechanism of pain relief by BTX is not completely understood. One thought is that the effect of BTX may extend further than physiologic muscle relaxation, inhibiting the release of neurotransmitters and neuropeptides that modulate pain. ${ }^{3}$ An additional hypothesis is that BTX may decrease the sympathetic transmission and hypersensitivity of the dorsal root ganglion, increasing the threshold for pain signaling. ${ }^{5}$ There are limited studies pertaining to treatment of chronic postoperative neck pain with BTX.

In 2010, Finiels and Batifol performed a preliminary study looking at the usefulness of BTX injections in managing chronic postoperative neck pain after cervical spine surgeries. Their study included 38 individuals with chronic postoperative neck pain who were divided equally into two groups. The first group (19 patients) received BTX injections, increasing in dosage from 20 to 100 units, every 3 months in the trapezius, supraspinatus, splenius capitis, and rhomboid muscles. The second group (19 patients) received a course of thiocolchicoside $16 \mathrm{mg}$, a muscle relaxant with anti-inflammatory and analgesic properties, and physical rehabilitation. The authors measured lordosis angles on lateral seated radiographs immediately after the procedure and again at 1.5 months after. An increase in these lordosis angles represents a decreased amount of muscular tension in key cervical muscles of the neck, as the increased tension on the cervical musculature flattens the lordotic curve. Visual analog scale (VAS) was also utilized to assess pain improvement. They found that $84 \%$ of the patients in the BTX group had increased postprocedure lordosis angles (an average of 11.3 degrees) in comparison to only $42 \%$ of the conservative group (an average of 4.7 degrees). In addition, the BTX group had a VAS score drop of 4.6 points as opposed to a decrease of only 0.6 points in the conservative group. The authors concluded that this preliminary study showed promising results and potential value in utilizing BTX injections for postoperative chronic neck pain. ${ }^{1}$

In 2014, Niezgoda et al described a case of a woman with severe postoperative neck and upper back pain after receiving spinal fusions of $\mathrm{C} 5$ to $\mathrm{C} 7$. After multiple failed conservative treatments, she was given BTX injections into the trapezius, masseter, sternocleidomastoids, scalenes, paraspinal, and digastric muscles. These BTX injections gave her pain relief for the first time since her spinal fusions. Combined with adjuvant pulsed electromagnetic field therapy, her pain had reduced to a more manageable level and she had additional improvements in her activities of daily living. ${ }^{3}$

In 2019, Finiels and Batifol returned with another study after having received the positive results from their 2010 study. This time the authors took a group of 43 patients who had chronic neck pain after cervical spine surgery (41 anterior interbody fusion, 1 laminoplasty, and 1 laminectomy) and used BTX-A injections on all 43 patients. Each participant received an average of 50 units (between 20 and 100 units) of BTX-A injections into their cervical musculature. Using the same scales as their previous study, both lordosis angles and VAS scores, they determined that favorable results for lordosis angulations would be a gain of at least 5 degrees and for VAS scores would be a decrease of at least two points. In their results, they stated that their participants demonstrated, on average, an increase in lordosis angles by 9.4 degrees and decrease in VAS scores of 2.5 points. According to their conclusions, 39 of the 43 (91\%) of their patients showed improvement in symptom. ${ }^{2}$

\section{Risks and Complications from Botulinum Toxin Use in the Neck}

The clinical use of neuromuscular toxins, particularly BTX, is not without risks. Even in successful cases of treating postoperative neck pain, expected nonemergent side effects may occur when utilizing BTX. ${ }^{3}$ Temporary muscle weakness may occur, considering that the primary effect of the neuromuscular toxin is muscle relaxation. Patients may experience focal muscle loss due to focal denervation at the neuromuscular junction. Focal muscle loss may present as changes in physical appearance, such as a narrowed neck or "scooped out" shoulder. ${ }^{3}$

One possible risk of repetitive BTX usage is the generation of anti-BTX antibodies, by the patient. These antibodies effectively block BTX and decrease the therapeutic effects of the toxin, limiting the benefits of subsequent injections. ${ }^{5}$ This occurs when the body is overexposed to BTX, primarily when an individual receives too many treatments in too short of an interval without the proper waiting period between procedures..$^{15}$ Experts agree that a fixed treatment interval of 12 weeks between BTX injections, regardless of the location of treatment, provides the best therapeutic results and usually avoids the creation of antibodies. Exposing the body to BTX injections more frequently and shorter than the 12-week interval increases the risk of antibody development and subsequent treatment failure. ${ }^{15}$

Chronic BTX usage can additionally cause a phenomenon known as dropped-head syndrome, a disabling condition caused by extreme weakness of the neck extensor musculature, leading to progressive cervical kyphosis and the inability to lift the head up against gravity. ${ }^{4,5,16}$ This complication can occur when BTX injections are applied to the muscles of neck extension. Hogan et al described a patient who had previously underwent C3 to C5 decompression and fusion, with rapidly progressing cervical kyphosis and severe neck weakness after receiving BTX-A 
"trigger point" injections into her neck extensor muscles for associated neck pain. She developed severe weakness of her cervical extensor muscles, leading to dropped-head syndrome. The authors determined that the semispinalis cervicis and semispinalis capitis muscles were primarily responsible for neck extension, so an extensive BTX blockade to these muscle bellies would prevent neck extension. ${ }^{5}$ In another study, Mohan and Chang highlighted a patient with a history of Arnold-Chiari type I malformation, congenital cervical canal stenosis, and previous C5 to C6 ACDF who presented with chronic postsurgical neck pain. They attempted to treat her neck pain with BTX, but their treatment exacerbated her acute-on-chronic canal stenosis, effectively causing increased neck pain and a transient dropped-head syndrome. With her avoidance of physical therapy, the authors postulated that her dropped-head syndrome was caused by biomechanical paraspinal instability and cervical hypermobility secondary to long-term BTX usage. ${ }^{4}$

At high doses, use of perioperative BTX can increase the risk of postoperative complications due to respiratory and swallowing difficulties, such as pneumonia. This results from neuromuscular blockade of muscles that are essential for normal respiration and swallowing. ${ }^{11}$ Racette et al described two cases of patients with dystonic cerebral palsy who received high doses of BTX-A into their neck musculature prior to undergoing surgical neck fixation. Patients 1 and 2 received 700 and 500 units, respectively, prior to their operations. While these high doses of BTX-A effectively stopped the cervical dystonia, permitting halo fixation and effective spinal fusion, both experienced postoperative complications. Patient 1 experienced vocal cord paralysis, difficulty swallowing, and resultant pooling of secretions. Patient 2 had epiglottic edema with poor airway protection, aspiration of gastric contents, and resultant aspiration pneumonia. As a result of these complications, both patients had placement of temporary gastrostomy and tracheostomy tubes. ${ }^{13}$ Despite the complications, they concluded that the procedure was a success because the spinal fusions held. ${ }^{13}$ These results presented by Racette et al may be limited to within the cerebral palsy population, but the general principles of management should still apply. When using BTX injections to the neck perioperatively, it is recommended to carefully monitor the respiratory and swallowing functions to decrease postoperative complications and morbidity. ${ }^{11}$

\section{Conclusion}

In conclusion, BTX has good clinical utility for cervical pain, spasms, and neuromuscular dysfunction (cervical dystonia, tics). In fact, $89 \%$ (56 out of 63) of patients who have been treated with BTX have had a decrease in their symptoms. ${ }^{1-3}$ Even with the observed benefits, it is important to remember that BTX usage is not without risk. The primary risks are mitigated by being judicious with the dosages of BTX and how often BTX is administered, as the adverse effects are primarily related to over administration., ${ }^{5,11,13,15}$ High doses of perioperative BTX can lead to postoperative swallowing, speaking, and breathing difficulties. ${ }^{11,13}$ Chronic administration can lead to muscle denervation and weakness, which can lead to more severe issues, such as "scooped out" shoulder, ${ }^{3}$ a narrowed neck, and dropped-head syndrome. ${ }^{4,5,16}$ Chronic usage can also lead to anti-BTX antibody formation, which blocks the action of BTX in the body. Proper monitoring of patients' responses to BTX and not administering it more often than every 12 weeks are the keys to avoiding these adverse effects. ${ }^{15}$ Additional studies, especially double-blinded randomized controlled trials, are needed to add further evidence for the efficacy of BTX in chronic postoperative neck pain; however, preliminary results have been promising. While one explanation is that the pain is alleviated by the BTX releasing the chronic tension, spasms, and rigidity that occur postoperatively after cervical fusions, other mechanisms including alterations of neurotransmitters, pain-modulating neuropeptides, sympathetic nerve transmissions, and dorsal root ganglions are being explored. ${ }^{3,5}$ Despite the risks and the incomplete understanding of the pain-relieving mechanisms of BTX, preliminary studies and selected cases have shown that BTX may have beneficial effects as a form of pain management for individuals with chronic neck pain after cervical spine surgeries. ${ }^{1-3}$

\section{Conflict of Interest}

None.

\section{References}

1 Finiels PJ, Batifol D. [Usefulness of botulinum toxin injections in the treatment of postoperative pain after cervical spine surgery: preliminary results]. Neurochirurgie 2010;56(5):374-381

2 Finiels PJ, Batifol D. The interest of botulinum toxin in the treatment of cervical pain of different origins. In: Neuropathic Pain. Avid Science; 2019:2-9

3 Niezgoda JA, Hardin ST, Kubat N, Acompanado J. The management of intractable pain with adjuvant pulsed electromagnetic field therapy. Adv Skin Wound Care 2014;27(5): 205-209

4 Mohan A, Chang E. Decompression for botulinum toxin-exacerbated cervical myeloradiculopathy in the setting of congenital stenosis and Arnold-Chiari I malformation. Spinal Cord Ser Cases 2018;4:42

5 Hogan KA, Manning EL, Glaser JA. Progressive cervical kyphosis associated with botulinum toxin injection. South Med J 2006;99(8):888-891

6 Ramachandran M,Eastwood DM. Botulinum toxin and its orthopaedic applications. J Bone Joint Surg Br 2006;88(8):981-987

7 Gooriah R, Fayyaz A. Therapeutic uses of botulinum toxin. J Clin Toxicol 2015;5(1):1-7

8 Adler $\mathrm{CH}$, Zimmerman RS, Lyons MK, Simeone F, Brin MF. Perioperative use of botulinum toxin for movement disorder-induced cervical spine disease. Mov Disord 1996;11(1): 79-81

9 Traynelis VC, Ryken T, Rodnitzky RL, Menezes AH. Botulinum toxin enhancement of postoperative immobilization in patients with cervical dystonia. Technical note. J Neurosurg 1992;77(5):808-809 
10 Iwamuro H, Takahashi H, Ide K, Nakauchi J, Taniguchi M. [Perioperative treatment with botulinum A toxin prior to posterior cervical decompression in a case with cervical spondylosis caused by spasmodic torticollis secondary to cerebral palsy]. No Shinkei Geka 2003;31(9):1015-1020

11 Kim KN, Ahn PG, Ryu MJ, et al. Long-term surgical outcomes of cervical myelopathy with athetoid cerebral palsy. Eur Spine J 2014;23(7):1464-1471

12 Furuya T, Yamazaki M, Okawa A, et al. Cervical myelopathy in patients with athetoid cerebral palsy. Spine 2013;38(3):E151-E157

13 Racette BA, Lauryssen C, Perlmutter JS. Preoperative treatment with botulinum toxin to facilitate cervical fusion in dystonic cerebral palsy. Report of two cases. J Neurosurg 1998;88(2):328-330

14 Takemoto M, Ikenaga M, Tanaka C, Sonobe M, Shikata J. Cervical dystonia induced by cervical spine surgery: a case report. Spine 2006;31(1):E31-E34

15 Escher CM, Paracka L, Dressler D, Kollewe K. Botulinum toxin in the management of chronic migraine: clinical evidence and experience. Ther Adv Neurol Disorder 2017;10(2):127-135

16 Rahimizadeh A, Soufiani HF, Rahimizadeh S. Cervical spondylotic myelopathy secondary to dropped head syndrome: report of a case and review of the literature. Case Rep Orthop 2016;2016:5247102 